\title{
A GAUSS-BONNET THEOREM FOR CONSTRUCTIBLE SHEAVES ON REDUCTIVE GROUPS
}

\author{
V. KIRITCHENKO
}

\section{Introduction}

In this paper, we prove an analog of the Gauss-Bonnet formula for constructible sheaves on reductive groups. This formula holds for all constructible sheaves equivariant under the adjoint action and expresses the Euler characteristic of a sheaf in terms of its characteristic cycle. As a corollary from this formula we get that if a perverse sheaf on a reductive group is equivariant under the adjoint action, then its Euler characteristic is nonnegative.

In the sequel by a constructible complex we will always mean a bounded complex of sheaves of $\mathbb{C}$-vector spaces whose cohomology sheaves are constructible with respect to some finite algebraic stratification.

We now formulate the main results. Let $G$ be a complex reductive group, and let $\mathcal{F}$ be a constructible complex on $G$. Denote by $C C(\mathcal{F})$ the characteristic cycle of $\mathcal{F}$. It is a linear combination of Lagrangian subvarieties $C C(\mathcal{F})=\sum c_{\alpha} \mathrm{T}_{X_{\alpha}}^{*} G$ (see [9]). Here and in the sequel $\mathrm{T}_{X}^{*} G$ denotes the closure of the conormal bundle to the smooth locus of a subvariety $X \subset G$. With $X$ one can associate a nonnegative number $\operatorname{gdeg}(X)$ called the Gaussian degree of $X$. It is equal to the number of zeros of a generic left-invariant differential 1-form on $G$ restricted to $X$. The precise definitions of the Gaussian degree and of the Gauss map are given in Section 2.

Theorem 1.1. If $\mathcal{F}$ is equivariant under the adjoint action of $G$, then its Euler characteristic can be computed in terms of the characteristic cycle by the following formula

$$
\chi(G, \mathcal{F})=\sum c_{\alpha} \operatorname{gdeg}\left(X_{\alpha}\right) .
$$

For a perverse sheaf the multiplicities $c_{\alpha}$ of its characteristic cycle are nonnegative [7]. The Gaussian degrees of $X_{\alpha}$ are also nonnegative by their definition, see below. Thus Theorem 1.1 immediately implies the following important corollary.

Corollary 1.2. If $\mathcal{F}$ is a perverse sheaf equivariant under the adjoint action of $G$, then its Euler characteristic is nonnegative.

Received May 9, 2002. 
In particular, let $\mathbb{C}_{X}$ be a constant sheaf on a subvariety $X \subset G$ extended by 0 to $G$. Applying the above statements to this sheaf we get the following corollary.

Corollary 1.3. If $X \subset G$ is a closed smooth subvariety invariant under the adjoint action of $G$, then $\chi(X)=(-1)^{\operatorname{dim} X} \operatorname{gdeg}(X)$. Thus the number $(-1)^{\operatorname{dim} X} \chi(X)$ is nonnegative.

Indeed, the characteristic cycle of $\mathbb{C}_{X}$ coincides with $(-1)^{\operatorname{dim} X} \mathrm{~T}_{X}^{*} G$. Corollary 1.3 is a noncompact analog of the classical Hopf theorem which states that the Euler characteristic of a compact oriented $C^{\infty}$-manifold $M$ is equal to $(-1)^{\operatorname{dim} M}$ times the number of zeros of a generic 1-form on $M$, counted with signs (coming from the orientation).

For the case when $G=\left(\mathbb{C}^{*}\right)^{n}$ is a torus, Corollary 1.2 was first proved by F. Loeser and C. Sabbah [11] with another proof given by O. Gabber and F. Loeser [6]. Theorem 1.1 was proved in the torus case by J. Franecki and M. Kapranov [4]. Theorem 1.1 holds for all constructible sheaves on a torus. However, it does not hold for arbitrary constructible sheaves on a noncommutative algebraic group (see [4] for a counterexample). M. Kapranov conjectured that it may be still true for constructible sheaves on reductive groups if we consider only sheaves equivariant under the adjoint action. The present paper proves this conjecture. Recently, A. Braverman proved nonnegativity of the Euler characteristic for some class of Ad $G$-equivariant $l$-adic sheaves in finite characteristic [1]. For complex ground field his result implies, in particular, Corollary 1.2 in the case, when a perverse sheaf coincides with its Goreski-MacPherson extension from the set of all regular semisimple elements of $G$. As the referee indicated the result of $O$. Gabber and F. Loeser holds for all holonomic $\mathcal{D}$-modules, and not only for those with regular singularities. The similar remark is valid for the A. Braverman's paper.

The main step in the proof of Theorem 1.1 is to reduce the problem to the case of a maximal torus $T \subset G$. Since $\mathcal{F}$ is $\mathrm{Ad} G$-equivariant, it is constructible with respect to some Whitney stratification $\mathcal{S}$ with Ad $G$-invariant strata. In Section 3 we prove that the Euler characteristic of a stratum $X \in \mathcal{S}$ coincides with that of the intersection $X \cap T$. This implies that the sheaf $\mathcal{F}$ restricted onto the maximal torus $T$ has the same Euler characteristic as $\mathcal{F}$. In Section 2 we recall some facts about Euler characteristic needed for the proof. In Section 4 we prove that the Gaussian degrees of $X$ and $X \cap T$ coincide.

To deal with the characteristic cycle we use the Dubson-Kashiwara index formula that expresses the multiplicities $c_{\alpha}$ in terms of the local Euler characteristic of $\mathcal{F}$ along each stratum and some topological data depending on the stratification only (Section 2). This data is given by the Euler characteristics with compact support of complex links. In our case we can choose a complex link to be invariant under the action of some compact torus and thus simplify computation of its Euler characteristic. This approach is taken from [2]. In Section 5 we prove that for any stratum $X_{\beta} \in \mathcal{S}$ and any semisimple stratum $X_{\alpha} \in \mathcal{S}$, such that $X_{\alpha} \subset \overline{X_{\beta}}$, the Euler characteristic with compact support of 
their complex link coincides with that of the complex link of the strata $X_{\alpha} \cap T$ and $X_{\beta} \cap T$. This allows us to view the formula from Theorem 1.1 as the same formula for the restriction of $\mathcal{F}$ onto $T$ (Section 6 ). Then we apply the result of $[4]$.

\section{Preliminaries}

Gaussian degree. We now define the (left) Gauss map and the Gaussian degree. The material of this subsection is taken from [4]. For more details see $[4,3]$.

Let $G$ be a complex algebraic group with Lie algebra $\mathfrak{g}$, and let $X$ be its subvariety of the dimension $k$. Denote by $\mathrm{G}(k, \mathfrak{g})$ the Grassmannian of $k$-dimensional subspaces in $\mathfrak{g}$. For any point $x \in G$, there is a natural isomorphism between the tangent space $\mathrm{T}_{x} G$ and $\mathfrak{g}$ given by the left multiplication by $x^{-1}$ :

$$
L_{x}: y \mapsto x^{-1} y ; \quad d_{x} L_{x}: \mathrm{T}_{x} G \rightarrow \mathfrak{g} .
$$

The left Gauss map $\Gamma_{X}: X \rightarrow \mathrm{G}(k, \mathfrak{g})$ is defined as follows:

$$
\Gamma_{X}(x)=d_{x} L_{x}\left(\mathrm{~T}_{x} X\right) .
$$

The Gauss map is rational and regular on the smooth locus $X^{s m}$ of $X$. If $X$ is a hypersurface, $\Gamma_{X}$ maps $X$ to $\mathbb{P}\left(\mathfrak{g}^{*}\right)$, which has the same dimension as $X$. In this case we define the Gaussian degree of $X$ to be the degree of its Gauss map. By the degree of a rational map $X \rightarrow Y$ we mean the number of preimages of a generic point in $Y$ (see [12], Proposition 3.17). In general case the Gaussian degree is the degree of a rational map $\tilde{\Gamma}_{X}: \tilde{X} \rightarrow \mathbb{P}\left(\mathfrak{g}^{*}\right)$, where $\tilde{X}$ and $\tilde{\Gamma}_{X}$ are defined as follows. The variety $\tilde{X}$ is a fiber bundle over $X^{s m}$, whose fiber at a point $x$ consists of all hyperplanes in $\mathfrak{g}$ that contain a subspace $\Gamma_{X}(x)$, i.e. $\tilde{X}=\left\{(x, y) \in X^{s m} \times \mathbb{P}\left(\mathfrak{g}^{*}\right): \Gamma_{X}(x) \subset y\right\}$. Then $\tilde{\Gamma}_{X}(x, y)=y$. Note that $\tilde{X}$ and $\mathbb{P}\left(\mathfrak{g}^{*}\right)$ have the same dimension. It is clear from the definition that the Gaussian degree is a birational invariant of a subvariety.

In the sequel we will use another description of the Gaussian degree. Let $\omega$ be a generic left-invariant differential 1-form on $G$ ( for reductive groups we define a generic 1-form explicitly in Section 4). We call a point $x \in X$ a zero of $\omega$, if $\omega$ restricted to the tangent space $\mathrm{T}_{x} X$ is zero. Then it is easy to verify that the Gaussian degree of $X$ is equal to the number of zeros of $\omega$ on $X$.

Euler characteristic. Let $T$ be a torus (it may be a complex torus $\left(\mathbb{C}^{*}\right)^{n}$ as well as a compact one $\left.\left(S^{1}\right)^{n}\right)$. Consider its linear algebraic action on $\mathbb{C}^{N}$, and a locally closed semialgebraic subset $X \subset \mathbb{C}^{N}$ invariant under this action. Let $X^{T} \subset X$ be the set of the fixed points. In what follows $\chi$ denotes the usual topological Euler characteristic and $\chi^{c}$ the Euler characteristic computed using cohomology with compact support. The following simple and well-known fact plays the crucial role in the sequel.

Proposition 2.1. The spaces $X$ and $X^{T}$ have the same Euler characteristic with compact support:

$$
\chi^{c}(X)=\chi^{c}\left(X^{T}\right)
$$


The following statement is also well-known, but the author could not find an appropriate reference.

Proposition 2.2. If $X$ is a complex algebraic variety, then $\chi^{c}(X)=\chi(X)$.

Proof. Applying Proposition 2.3 to the constant sheaf $\mathbb{C}_{X}$ and using additivity of the Euler characteristic with compact support, one can deduce this equality from the following fact. For any point $x \in X$ the Euler characteristic with compact support of a small open neighborhood of $x$ is equal to 1 . To prove this fact we use the induction by the dimension of $X$.

We may assume that a neighborhood of $x$ is embedded in $\mathbb{C}^{N}$. Take a generic holomorphic function $f$ on $X$ such that $f(x)=0$, and an open neighborhood $C=f^{-1}(D) \cap B$, where $D \subset \mathbb{C}$ is a small open disk with the center at 0 and $B \subset \mathbb{C}^{N}$ is a small ball with the center at $x$. Then $f: C \backslash f^{-1}(0) \rightarrow D \backslash\{0\}$ is a fiber bundle (see [8], Section 2.4). Thus $\chi^{c}\left(C \backslash f^{-1}(0)\right)=0$, and $\chi^{c}(C)=$ $\chi^{c}\left(f^{-1}(0)\right)$. The dimension of $f^{-1}(0)$ is already less than that of $X$.

The Euler characteristic of sheaves. We now recall a formula for the Euler characteristic of constructible sheaves on varieties. Let $X \subset \mathbb{C}^{N}$ be a smooth subvariety, and let $\mathcal{F}$ be a constructible complex on $X$. With any point $x \in X$ one can associate the local Euler characteristic $\chi\left(\mathcal{F}_{x}\right)$ of $\mathcal{F}$ at this point. Thus $\mathcal{F}$ gives rise to the constructible function $\chi(\mathcal{F})$ on $X$ by the formula $\chi(\mathcal{F})(x)=$ $\chi\left(\mathcal{F}_{x}\right)$.

There is the concept of the direct image of a constructible function (see [5] and [9], Section 9.7). It is defined for any morphism of algebraic varieties $X \rightarrow Y$ and a constructible function on $X$. We use the more suggestive notation $\int_{X} f(x) d \chi$ for the direct image of $f$ under the morphism $X \rightarrow p t$, since this direct image may be also defined as the integral of $f$ over the Euler characteristic $[13,10]$.

Proposition 2.3. The global Euler characteristic $\chi(X, \mathcal{F})=\sum(-1)^{i} H^{i}(X, \mathcal{F})$ is equal to the following integral over the Euler characteristic

$$
\chi(X, \mathcal{F})=\int_{X} \chi(\mathcal{F}) d \chi
$$

In other words, if we fix a finite algebraic stratification $X=\bigsqcup X_{\alpha}, \alpha \in \mathcal{S}$, such that the function $\chi(\mathcal{F})$ is constant along each stratum, we get

$$
\chi(X, \mathcal{F})=\sum_{\alpha \in \mathcal{S}} \chi_{\alpha}(\mathcal{F}) \chi^{c}\left(X_{\alpha}\right),
$$

where $\chi_{\alpha}(\mathcal{F})$ is the value of $\chi(\mathcal{F})$ at any point of a stratum $X_{\alpha}$.

See [9], Section 9.7 for the proof.

Complex links and characteristic cycles. We use the notation of the previous subsection. Suppose that $\mathcal{S}$ is a Whitney stratification of $X$. Let $X_{\alpha}, X_{\beta}, \alpha, \beta \in$ $\mathcal{S}$, be two strata such that $X_{\alpha} \subset \overline{X_{\beta}}$. Choose a point $a \in X_{\alpha}$ and any normal slice $N \subset X$ to $X_{\alpha}$ at the point $a$. Consider a holomorphic function $l$ on $N$ such that $l(a)=0$ and its differential $d_{a} l$ is a generic covector in the 
cotangent space $\mathrm{T}_{a}^{*} N$ (i.e. $d_{a} l$ belongs to some open dense subset of this space that depends on the stratification $\mathcal{S})$. Let $h(\cdot, \cdot)$ be a Hermitian metric in $\mathbb{C}^{N}$ and $B=\left\{x \in \mathbb{C}^{N}: h(x-a, x-a) \leq\right.$ const $\}$ a small ball with the center at $a$.

We now define the complex link $L$ of the strata $X_{\alpha}, X_{\beta}$ as $L=B \cap l^{-1}(\varepsilon) \cap X_{\beta}$. If the absolute value of $\varepsilon$ and the radius of the ball $B$ are small enough, the result up to a homeomorphism does not depend on any of the choices involved (see [8], Section 2.3 for the proof $)$. We will use the notation $\mathrm{e}(\alpha, \beta)$ as well as $\mathrm{e}\left(X_{\alpha}, X_{\beta}\right)$ for the Euler characteristic of $L$ with compact support. We also set e $(\alpha, \alpha)=-1$.

The numbers $\mathrm{e}(\alpha, \beta)$ are useful when one need to find the multiplicities of the characteristic cycle $C C(\mathcal{F})$. Multiplicities are recovered from the constructible function $\chi(\mathcal{F})$ by the following theorem of Dubson and Kashiwara.

Theorem 2.4. The characteristic cycle of $\mathcal{F}$ is the linear combination of Lagrangian subvarieties $\mathrm{T}_{X_{\alpha}}^{*} X, \alpha \in \mathcal{S}$, with coefficients

$$
c_{\alpha}=(-1)^{\operatorname{dim} X_{\alpha}+1} \sum_{X_{\alpha} \subset \overline{X_{\beta}}} \mathrm{e}(\alpha, \beta) \chi_{\beta}(\mathcal{F}) .
$$

See [7], Theorem 8.2 for the proof.

\section{Euler characteristic of invariant subvarieties}

Let $G$ be a connected reductive group over $\mathbb{C}$, and $T$ a maximal complex torus in $G$. Consider a subvariety $X \subset G$ invariant under the adjoint action of $G$.

Proposition 3.1. The varieties $X$ and $X \cap T$ have the same Euler characteristic with compact support. Moreover, $\chi(X)=\chi(X \cap T)$.

Proof. The subvariety $X$ is invariant under the adjoint action of $G$. In particular, it is invariant under the adjoint action of the maximal torus $T$. The set $G^{T} \subset G$ of the fixed points under this action coincides with $T$, since the centralizer of the maximal torus coincides with the maximal torus itself. Thus by Proposition 2.1 the varieties $X$ and $X \cap T$ have the same Euler characteristic with compact support. Combining this result with Proposition 2.2, we get that $\chi(X)=$ $\chi(X \cap T)$.

Example. Let $X=\mathcal{O}_{a}$ be the orbit of an element $a \in G$ under the adjoint action of $G$. Then Proposition 3.1 implies that if $a$ is semisimple, then $\chi\left(\mathcal{O}_{a}\right)$ is equal to the number $\left|\mathcal{O}_{a} \cap T\right|$ of intersection points. We may choose the maximal torus $T$ such that $a \in T$. Since the orbit of $a$ under the action of the Weyl group $W$ on $T$ coincides with $\mathcal{O}_{a} \cap T$, we obtain that $\chi\left(\mathcal{O}_{a}\right)=|W| / \mid$ Stab $a \mid$, where Stab $a \subset W$ is the stabilizer of $a$ in $W$. If $a$ is not semisimple, then $\chi\left(\mathcal{O}_{a}\right)=0$.

Let $\mathcal{F}$ be a constructible complex on $G$.

Proposition 3.2. Suppose that $\mathcal{F}$ is equivariant under the adjoint action of $G$. Let $\mathcal{F}_{T}$ be a restriction of $\mathcal{F}$ onto $T \subset G$. Then the sheaves $\mathcal{F}$ and $\mathcal{F}_{T}$ have the same Euler characteristic:

$$
\chi(G, \mathcal{F})=\chi\left(T, \mathcal{F}_{T}\right) .
$$


Proof. The sheaves $\mathcal{F}$ and $\mathcal{F}_{T}$ have the same local Euler characteristic at a point $x \in T$, since $\mathcal{F}_{T}$ is the restriction of $\mathcal{F}$ onto $T$. Thus the Euler characteristic $\chi\left(T, \mathcal{F}_{T}\right)$ is equal to $\int_{T} \chi(\mathcal{F}) d \chi$ by Proposition 2.3. The function $\chi(\mathcal{F})$ is invariant under the adjoint action of $G$, and Proposition 3.1 implies

$$
\int_{T} \chi(\mathcal{F}) d \chi=\int_{G} \chi(\mathcal{F}) d \chi
$$

The last integral is equal to the Euler characteristic $\chi(G, \mathcal{F})$ by Proposition 2.3 .

\section{Gaussian degree of invariant subvarieties}

We now compare the Gaussian degrees of $X$ in $G$ and of $X \cap T$ in $T$. Clearly, the Gaussian degree of a $k$-dimensional subvariety is equal to the sum of the Gaussian degrees of its $k$-dimensional irreducible components. Thus we may assume that $X$ is irreducible. There are two cases: the set of all nonsemisimple elements of $X$ has codimension either 0 or at least 1 . In what follows we prove that in the first case $\operatorname{gdeg}(X)=0$, and in the second case $\operatorname{gdeg}(X)=\operatorname{gdeg}(X \cap$ $T$ ). In particular, the Gaussian degree of any orbit $\mathcal{O}_{a} \subset G$ coincides with the Gaussian degree of its intersection with the maximal torus $T$.

Any reductive group $G$ admits an embedding in $\mathrm{GL}_{N}(\mathbb{C})$ for some $N$, such that the inner product $\operatorname{tr}\left(Y_{1} Y_{2}\right)$ is nondegenerate on Lie algebra $\mathfrak{g}$. Let us fix such an embedding. Then $\mathfrak{g}$ may be identified with the space of all left-invariant differential 1-forms on $G$ : an element $S \in \mathfrak{g}$ gives rise to a 1 -form $\omega$ by the formula

$$
\omega(Y)=\operatorname{tr}\left(x^{-1} Y S\right)
$$

where $x \in G$ and $Y \in \mathrm{T}_{x} G$. We will call such a form generic, if $S$ is regular semisimple.

Lemma 4.1. All generic left invariant 1-forms form an open dense subset in the space of all left-invariant 1-forms.

Proof. All regular semisimple elements form an open dense subset in $\mathfrak{g}$. This implies the statement of the lemma.

Proposition 4.2. The Gaussian degree of an orbit $\mathcal{O}_{a}$ is equal to the number of the intersection points $\mathcal{O}_{a} \cap T$. In particular, if a is a nonsemisimple element, then $\operatorname{gdeg}\left(\mathcal{O}_{a}\right)=0$.

Proof. Consider the map

$$
\varphi: G \rightarrow \mathcal{O}_{a} ; \quad \varphi: g \mapsto g a g^{-1} .
$$

Since $\varphi$ is smooth and surjective, the tangent space $\mathrm{T}_{x} \mathcal{O}_{a}$ is the image of the induced map $d \varphi$. A simple computation shows that $\mathrm{T}_{x} \mathcal{O}_{a}=[\mathfrak{g}, x]$. Let $\omega$ be a generic left-invariant differential 1-form on $G$ given by the formula (1). Then $\omega=0$ on $\mathrm{T}_{x} \mathcal{O}_{a}$ is equivalent to $\operatorname{tr}\left(x^{-1} Y x S-Y S\right)=0$ for any $Y \in \mathfrak{g}$. Since the form $\operatorname{tr}\left(Y_{1} Y_{2}\right)$ is Ad $G$-invariant, we have $\operatorname{tr}\left(x^{-1} Y x S-Y S\right)=\operatorname{tr}\left(Y\left(x S x^{-1}-S\right)\right)$. 
The form $\operatorname{tr}\left(Y_{1} Y_{2}\right)$ is nondegenerate on $\mathfrak{g}$. Thus $x$ and $S$ commute, and $x$ belongs to some maximal torus $T_{S}$ that depends on $S$.

Remark. Suppose that an element a lies in the maximal torus $T$. Then the space $\mathrm{T}_{a} \mathcal{O}_{a}=[\mathfrak{g}, a]$ is orthogonal to the tangent space $\mathrm{T}_{a} T$ with respect to the form $\left(Y_{1}, Y_{2}\right) \mapsto \operatorname{tr}\left(a^{-1} Y_{1} \cdot a^{-1} Y_{2}\right)$. Since this form is nondegenerate on $\mathrm{T}_{a} T$, we get that at any point $x \in \mathcal{O}_{a} \cap T$ the intersection of tangent spaces $\mathrm{T}_{x} \mathcal{O}_{a}$ and $\mathrm{T}_{x} T$ is zero.

Corollary 4.3. Let $Z \subset G$ be an irreducible subvariety invariant under the adjoint action of $G$, such that the set $Z_{n}$ of all nonsemisimple elements of $Z$ is a Zariski open nonempty subset in $Z$. Then $\operatorname{gdeg}(Z)=0$.

Proof. The Gaussian degree of a subvariety $Z \subset G$ is birationally invariant. Therefore, it suffices to compute it for $Z_{n}$. Let $\omega$ be a generic left-invariant differential 1-form on $G$ given by the formula (1). For any smooth point $a \in Z_{n}$ the restriction of this form on the subspace $\mathrm{T}_{a} \mathcal{O}_{a} \subset \mathrm{T}_{a} Z_{n}$ of the tangent space $\mathrm{T}_{a} Z_{n}$ is already nonzero by Proposition 4.2. Thus the form $\omega$ does not vanish in any smooth point of $Z_{n}$, and $\operatorname{gdeg}(Z)=\operatorname{gdeg}\left(Z_{n}\right)=0$.

Proposition 4.4. Let $X$ be an irreducible subvariety of $G$ invariant under the adjoint action of $G$ such that the subset of all nonsemisimple elements of $X$ has codimension at least 1 in $X$. Then the Gaussian degrees of $X$ in $G$ and of $X \cap T$ in $T$ coincide.

Proof. Let $k$ be the maximal dimension of a semisimple orbit in X. Denote by $X_{s}$ the set of all semisimple elements in $X$, whose orbits have dimension $k$. Then $X_{s}$ is a Zariski open subset of $X$. Consider the map

$$
\varphi: G \times(X \cap T) \rightarrow X, \quad(g, t) \mapsto g t g^{-1} .
$$

The image of $\varphi$ contains $X_{s}$. Since $X_{s}$ is a Zariski open nonempty subset of $X$, $\operatorname{gdeg}(X)=\operatorname{gdeg}\left(X_{s}\right)$. For any smooth point $x \in X_{s}$ the tangent space $\mathrm{T}_{x} X$ is again the image of the induced map

$$
d \varphi: \mathrm{T}_{g} G \times \mathrm{T}_{t}(X \cap T) \rightarrow \mathrm{T}_{x} X,
$$

where $g t g^{-1}=x$. By calculating $d \varphi$ we obtain that $\mathrm{T}_{x} X=[\mathfrak{g}, x] \oplus g \mathrm{~T}_{t}(X \cap$ $T) g^{-1}$. Let $\omega$ be a generic left-invariant differential 1-form on $G$ given by the formula (1). Then $\omega=0$ on $\mathrm{T}_{x} X$ is equivalent to $\omega=0$ on $[\mathfrak{g}, x]$ and $\omega=0$ on $g \mathrm{~T}_{t}(X \cap T) g^{-1}$. The first identity holds if and only if $x$ belongs to the maximal torus $T_{S}$ (see the proof of Proposition 4.2). Denote by $\omega_{T}$ the restriction of $\omega$ on $T^{*} T_{S}$. If $x \in T_{S}$, then $g \mathrm{~T}_{t}(X \cap T) g^{-1}=\mathrm{T}_{x}\left(X \cap T_{S}\right)$. Thus the form $\omega$ vanishes on $\mathrm{T}_{x} X$ if and only if the form $\omega_{T}$ vanishes on $\mathrm{T}_{x}\left(X \cap T_{S}\right)$. It follows that $\operatorname{gdeg}(X)=\operatorname{gdeg}\left(X \cap T_{S}\right)=\operatorname{gdeg}(X \cap T)$, since all maximal tori are conjugate. 


\section{The Euler characteristic of the complex link}

We now compute the Euler characteristic with compact support of a complex link for a certain class of stratifications of $G$. For any $a \in G$ we define the rank of $a$ to be the dimension of its centralizer in $G$. A Whitney stratification $\mathcal{S}$ of $G$ is called admissible if the following conditions hold. For every $\alpha \in \mathcal{S}$

- the stratum $X_{\alpha}$ is invariant under the adjoint action of $G$,

- elements of $X_{\alpha}$ are either all semisimple of the fixed rank or all nonsemisimple.

Denote by $\mathcal{S}_{0} \subset \mathcal{S}$ the subset of all semisimple strata. Due to the second condition and the Remark after Proposition 4.2, for any semisimple stratum $X_{\alpha} \in \mathcal{S}$ intersection $X_{\alpha} \cap T$ is smooth, and at any point $x \in X_{\alpha} \cap T$ the intersection of the tangent spaces $\mathrm{T}_{x} X_{\alpha} \cap \mathrm{T}_{x} T$ coincides with the tangent space $\mathrm{T}_{x}\left(X_{\alpha} \cap T\right)$. Thus we can consider an induced Whitney stratification $\mathcal{S}_{T}$ of the maximal torus $T$, namely, $T=\bigsqcup\left(X_{\alpha} \cap T\right), \alpha \in \mathcal{S}_{0}$.

Proposition 5.1. Consider two strata $X_{\alpha}$ and $X_{\beta}$ such that $X_{\alpha}$ belongs to the closure of $X_{\beta}$. If $X_{\alpha}$ is semisimple and $X_{\beta}$ is not, then $\mathrm{e}(\alpha, \beta)=0$. If both $X_{\alpha}$, $X_{\beta}$ are semisimple, then $\mathrm{e}(\alpha, \beta)=\mathrm{e}\left(X_{\alpha} \cap T, X_{\beta} \cap T\right)$, where the complex link of $X_{\alpha} \cap T$ and $X_{\beta} \cap T$ is taken in the torus $T$.

Proof. Let $Z \subset G$ be the centralizer of an element $a \in X_{\alpha}$. Then $Z$ is again a reductive group. Since the tangent spaces $\mathrm{T}_{a} Z$ and $\mathrm{T}_{a} \mathcal{O}_{a}$ are orthogonal with respect to the form $\left(Y_{1}, Y_{2}\right) \mapsto \operatorname{tr}\left(a^{-1} Y_{1} \cdot a^{-1} Y_{2}\right)$, and this form is nondegenerate on $\mathrm{T}_{a} Z$, we get that $Z$ is the normal slice to the orbit $\mathcal{O}_{a} \subset X_{\alpha}$. Thus any normal slice to $X_{\alpha} \cap Z$ in $Z$ will also be the normal slice to $X_{\alpha}$ in $G$. Let us construct a normal slice $N \subset Z$ invariant under the adjoint action of $Z$.

Let $k$ be the dimension of $X_{\alpha} \cap Z$. Some neighborhood of $a$ in $X_{\alpha} \cap Z$ lies in the center of $Z$, because all elements of $X_{\alpha}$ have the same rank. Thus we can find $k$ characters $\varphi_{1}, \ldots, \varphi_{k}$ of the group $Z$ such that their differentials $d_{a} \varphi_{1}, \ldots, d_{a} \varphi_{k}$ restricted to the tangent space $\mathrm{T}_{a}\left(X_{\alpha} \cap Z\right)$ are linearly independent. Let $N \subset Z$ be the set of common zeros of the system $\varphi_{1}\left(z a^{-1}\right)=\ldots=\varphi_{k}\left(z a^{-1}\right)=1$.

\section{Example.}

a) Let $G$ be $\mathrm{GL}_{N}(\mathbb{C})$ and let $X_{\alpha}=Z\left(\mathrm{GL}_{N}\right)=\mathbb{C}^{*}$ be the center of $\mathrm{GL}_{N}$. Then $Z=G$, and the only characters of $Z$ are the powers of determinant. We have $d_{e}$ det $=\operatorname{tr}$ for the identity element $e \in \mathrm{GL}_{N}$, and $\operatorname{tr}$ is a nonzero linear function on $\mathbb{C}^{*} e$. Thus at the point $e \in X_{\alpha}$ we can take $N=\mathrm{SL}_{N}(\mathbb{C})$.

b) Let $G$ be any reductive group, and let $X_{\alpha}$ be a stratum consisting of regular semisimple elements. Then $Z$ is a maximal torus. Thus any normal slice to $X_{\alpha} \cap Z$ in $Z$ is invariant under the adjoint action of $Z$.

We now continue the proof of Proposition 5.1. Consider a generic linear function $l$ on $N$ given by the formula $l(x)=\operatorname{tr}\left(\left(a^{-1} x-e\right) S\right)$, where $S \in$ Lie $Z$ is regular semisimple. There exists a maximal torus $T \subset Z$ centralizing $S$. Since $a$ is semisimple, $T$ is also a maximal torus in $G$. For any $\varepsilon$ the set $l^{-1}(\varepsilon)$ is invariant under the adjoint action of $T$. Denote by $T_{c}$ the compact form of $T$. 
Choose a Hermitian inner product $h(\cdot, \cdot)$ on $\mathfrak{g l}_{N}$ invariant under the adjoint action of $T_{c}$ and a small ball $B=\left\{x \in \mathfrak{g l}_{N}: h(x-a, x-a) \leq\right.$ const $\}$.

Thus with a generic vector $S \in$ Lie $Z$ we associate the complex link $L=$ $B \cap l^{-1}(\varepsilon) \cap X_{\beta}$ of the strata $X_{\alpha}$ and $X_{\beta}$. The complex link $L$ is invariant under the adjoint action of the torus $T_{c}$ by the construction. Thus by Proposition 3.1 we get $\chi^{c}(L)=\chi^{c}\left(L^{T_{c}}\right)=\chi^{c}\left(L \cap Z^{T_{c}}\right)$. Note that $Z^{T_{c}}=Z^{T}=T$. If $X_{\beta}$ is nonsemisimple, $X_{\beta} \cap T$ is empty, thus $L \cap T$ is empty. It follows that $\mathrm{e}(\alpha, \beta)=0$ in this case. If $X_{\beta}$ is semisimple, then $L \cap T$ is a complex link for $X_{\alpha} \cap T, X_{\beta} \cap T$ in the torus $T$.

Corollary 5.2. If $X$ is a smooth irreducible subvariety invariant under the adjoint action of $G$, then either $X$ consists of nonsemisimple elements only or the set of all semisimple elements in $X$ is dense. In particular, if in addition $X$ is closed, then it contains a dense subset of semisimple elements. The Gaussian degrees of $X$ and of $X \cap T$ coincide.

Proof. Let us prove the first statement by contradiction. Let $\mathcal{S}$ be an admissible stratification of $G$ subordinate to $X$, and let $X_{n} \subset X$ be a maximal open stratum in $X$, such that $X_{n}$ is nonsemisimple. Then $\operatorname{dim} X_{n}=\operatorname{dim} X$, and $X-X_{n}$ contains at least one semisimple stratum $X_{s}$. The number e $\left(X_{s}, X_{n}\right)$ is zero by Proposition 5.1. That contradicts to the smoothness of $X$. Combining the first statement with the results of Section 4, we get the last statement.

\section{Proof of Theorem 1.1}

Since $\mathcal{F}$ is constructible and equivariant under the adjoint action, there exists some finite algebraic Whitney stratification $\mathcal{S}$ subordinate to $\mathcal{F}$ such that each stratum is invariant under the adjoint action. Stratifying each stratum if necessary we may assume that $\mathcal{S}$ is admissible. Let us apply Theorem 2.4 and Proposition 5.1 to the characteristic cycles of $\mathcal{F}$ and of $\mathcal{F}_{T}$. Notice that for a semisimple stratum $X_{\alpha} \in \mathcal{S}$ the difference $\operatorname{dim} X_{\alpha}-\operatorname{dim}\left(X_{\alpha} \cap \mathcal{S}\right)$ is equal to $\operatorname{dim} \mathcal{O}_{a}, a \in X_{\alpha}$, and the latter is even. As a straightforward corollary we get

Corollary 6.1. Let $X_{\alpha} \in \mathcal{S}$ be a semisimple stratum. The multiplicities of characteristic cycles of $\mathcal{F}$ and $\mathcal{F}_{T}$ along the strata $X_{\alpha}$ and $X_{\alpha} \cap T$, respectively, coincide.

Example. Suppose that the support of the constructible function $\chi(\mathcal{F})$ lies in the closure of an orbit $\mathcal{O}_{a}, a \in G$. This kind of sheaves is studied in [2] for unipotent orbits. In this case the strata of an admissible stratification that contribute to the characteristic cycle are the orbits in $\overline{\mathcal{O}_{a}}$. Let $a_{s}, a_{n} \in G$ be the semisimple and unipotent elements respectively such that $a=a_{s} \cdot a_{n}$. Then $X_{\alpha}=\mathcal{O}_{a_{s}}$ is the only semisimple stratum in $\overline{\mathcal{O}_{a}}$. Thus for the multiplicity $c_{\alpha}(\mathcal{F})$ of $C C(\mathcal{F})$ along this stratum we get $c_{\alpha}(\mathcal{F})=\chi_{\alpha}(\mathcal{F})=c_{\alpha}\left(\mathcal{F}_{T}\right)$.

Now the formula of Theorem 1.1 reduces to the same formula for the sheaf $\mathcal{F}_{T}$ and the stratification $\mathcal{S}_{T}$. First, $\chi(\mathcal{F}, G)=\chi\left(\mathcal{F}_{T}, T\right)$ by Proposition 3.2. Second, for all nonsemisimple strata $X_{\alpha}, \alpha \in \mathcal{S}$, we have $\operatorname{gdeg}\left(X_{\alpha}\right)=0$ by Corollary 4.3. 
Thus the right hand side of the formula may be considered as the sum over semisimple strata only, i.e.

$$
\chi(X, \mathcal{F})=\sum_{\alpha \in \mathcal{S}_{0}} c_{\alpha}(\mathcal{F}) \operatorname{gdeg}\left(X_{\alpha}\right) .
$$

By Corollary 6.1 this is equivalent to the formula

$$
\chi\left(T, \mathcal{F}_{T}\right)=\sum_{\alpha \in \mathcal{S}_{T}} c_{\alpha}\left(\mathcal{F}_{T}\right) \operatorname{gdeg}\left(X_{\alpha} \cap T\right),
$$

since $\operatorname{gdeg}\left(X_{\alpha}\right)=\operatorname{gdeg}\left(X_{\alpha} \cap T\right)$ by Proposition 4.4. To prove the latter formula we apply Theorem 1.3 from [4].

\section{Acknowledgements}

I am grateful to M. Kapranov for constant encouragement, valuable suggestions and remarks. I would also like to thank A. Braverman and A. Khovanskii for useful discussions.

\section{References}

[1] A. Braverman, On Euler characteristic of equivariant sheaves, preprint: http://xxx.lanl.gov/abs/math.AG/0202165

[2] S. Evens, I. Mirković, Characteristic cycles for the loop Grassmannian and nilpotent orbits, Duke Math. J. 97 (1999), 109-126.

[3] J. Franecki, The Gauss map and Euler characteristic on algebraic groups, PhD thesis, Northwestern University, Evanston, Illinois, 1998.

[4] J. Franecki, M. Kapranov, The Gauss map and a noncompact Riemann-Roch formula for constructible sheaves on semiabelian varieties, Duke Math. J. 104 (2000), 171-180.

[5] W. Fulton, R. MacPherson, Categorical framework for the study of singular spaces, Mem. Amer. Math. Soc. 31 (1981), no. 243.

[6] O. Gabber, F. Loeser, Faisceaux pervers l-adiques sur un tore, Duke Math. J. 83 (1996), 501-606.

[7] V. Ginsburg, Characteristic varieties and vanishing cycles, Invent. Math. 84 (1986), 327402.

[8] M. Goresky, R. MacPherson, Stratified Morse theory, Ergebnisse der Mathematik und ihrer Grenzgebiete (3), 14. Springer-Verlag, Berlin, 1988.

[9] M. Kashiwara, P. Schapira, Sheaves on manifolds, Grundlehren der Mathematischen Wissenschaften, 292. Springer-Verlag, Berlin, 1990.

[10] A. Khovanskii, A. Pukhlikov, Integral transforms based on Euler charcteristic and their applications, Integral Transform. Spec. Funct. 1 (1993), 19-26.

[11] F. Loeser, C. Sabbah, Caractérisation des D-modules hypergéométriques irréductibles sur le tore. I, C. R. Acad. Sci. Paris Sér. I Math. 312 (1991), 735-738; II, 315 (1992), 1263-1264.

[12] D. Mumford, Algebraic geometry. I. Complex projective varieties, Springer, 1976.

[13] O. Viro, Some integral calculus based on Euler characteristic, Topology and geometryRohlin Seminar, 127-138, Lecture Notes in Math., 1346, Springer, Berlin, 1988.

Department of Mathematics, University of Toronto, Toronto, Ontario, Canada. M5S 3 G3.

E-mail address: vkiritch@math.toronto.edu 